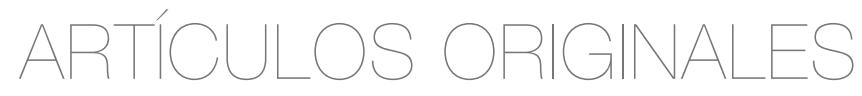

\title{
Ley Orgánica de Precios Justos y la fijación de precios en las ferreterías del municipio Campo Elías, Estado Mérida, Venezuela
}

\author{
JUAN CARLOS GARCÍA CONTRERAS* \\ MARYSELA COROMOTO MORILLO MORENO**
}

* MSc. en Administración, Mención Finanzas. Universidad de Los Andes, Mérida, Venezuela. E-mail: juanypunto@hotmail.com. ORCID: 0000-0001-5590-9690. Google Scholar: https://scholar.google.com/ citations?hl=es\&user=xOXhoroAAAAJ.

** Doctora en Ciencias Económicas y Empresariales. Profesora Titular Universidad de Los Andes, Mérida, Venezuela. E-mail: marysela.morillo@gmail.com. ORCID: 0000-0003-0697-4677. Google Scholar: https://scholar.google.com/ citations? user=DsJhEwYAAAAJ\&hl=es. 


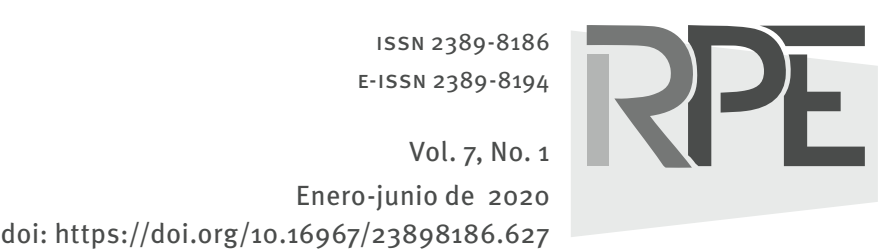

COMO CITAR ESTE ARTÍCULO

How to cite this article:

García, J.C. y Morillo, M. (2020). Ley Orgánica de Precios Justos y la fijación de precios en las ferreterías del municipio Campo Elías, Estado Mérida, Venezuela. Revista Perspectiva Empresarial, 7(1), 47-59.

Recibido: 03 de noviembre de 2019

Aprobado: 22 de enero de 2020
RESUMEN Con el propósito de evaluar el efecto de la fijación de precios en la rentabilidad de las ferreterías del municipio Campo Elías del Estado Mérida (Venezuela), en el marco de la vigente Ley Orgánica de Precios Justos, se diseñó un estudio de tipo analítico no experimental - con base en un censo- para estudiar la totalidad de ferreterías activas al momento de la recolección de datos. Con una tasa de respuesta del $40 \%$ se encontró que los conceptos considerados como costo del producto vendido al momento de fijar los precios son costos según factura de la mercancía comprada, seguido de los impuestos no recuperables o transferibles al consumidor y los costos de transporte, fletes o traslado, omitiendo aranceles y gastos de importación (aduanas). Más del $50 \%$ de los precios de los artículos ofertados no son fijados y marcados o etiquetados por su proveedor, lo cual representa un incumplimiento legal en el marcaje de precios de venta al público; agravado con el riesgo de remoción de precios por parte de los integrantes de la cadena de comercialización. Finalmente se recomiendan mayores actividades de fiscalización por parte de los entes competentes.

PALABRAS CLAVE costos, contabilidad, gerencia, precios y rentabilidad.

\section{Organic Law of Fair Prices and price fixation in hardware stores of the Campo Elías municipality, Mérida State, Venezuela}

ABSTRACT In the framework of the current Organic Law of Fair Prices and with the aim of evaluating the effect of price fixation on the profitability of hardware stores of the Campo Elías municipality in the Mérida State (Venezuela), an analytical nonexperimental study was designed - based on a census - in order to study the total number of hardware stores active at the time data was collected. With a response rate of $40 \%$, it was found that the concepts considered as the cost of product sold when fixing prices are those costs as per invoice of the purchased merchandise, followed by taxes non-recoverable or non-transferable to the consumer, and transport, freight or movement costs, omitting import duties and import expenses (customs). More than $50 \%$ of the prices of offered items are not fixed, marked or labeled by the supplier, which represents a legal breach in labeling selling prices for the public. This is made more serious with the risk of price removal by members of the commercialization chain. Finally, more fiscal activities by regulatory entities are recommended.

KEY WORDS costs, accounting, management, prices and profitability. 


\section{Empregabilidade interna percebida como estratégia para reduzir a disposição a renunciar dos funcionários}

RESUMO Com o objetivo de avaliar o efeito da precificação sobre a rentabilidade das lojas de ferragens no município de Campo Elías, no Estado de Mérida (Venezuela), no âmbito da Lei Orgânica sobre Preços Justos, foi elaborado um estudo analítico não experimental -com base em um censo- para estudar todas as lojas de hardware ativas no momento da coleta de dados. Com uma taxa de resposta de $40 \%$, verificou-se que os conceitos considerados como o custo do produto vendido no momento da fixação dos preços são custos de acordo com a fatura da mercadoria comprada, seguidos de impostos não recuperáveis ou transferíveis para os custos de consumo e transporte, frete ou transferência, omitindo direitos e despesas de importação (alfândega). Mais de $50 \%$ dos preços dos itens oferecidos não são definidos e marcados ou rotulados pelo seu fornecedor, o que representa uma violação legal na marcação dos preços de varejo; agravada pelo risco de remoção de preços por membros da cadeia de marketing. Por fim, são recomendadas maiores atividades de inspeção pelas entidades competentes.

PALAVRAS-CHAVE custos, contabilidade, administração, preços e rentabilidade. 


\section{Introducción}

Hoy a nivel mundial es habitual el progreso de las organizaciones con fines de lucro en diversos sectores de la economía, siendo compañías que se esmeran en obtener el punto óptimo entre niveles de costos mínimos y la máxima calidad en la entrega de su producto o servicio. La globalización de la economía conlleva a la exigente competencia entre empresas en diversos países, por lo que son presionadas por los mercados para renovar y aumentar sus capacidades operativas; sirviendo esto como desafío para que las empresas - tanto productivas como de serviciosse esfuercen en mantener determinados niveles de sustentabilidad o al menos ser empresas sostenibles con resultados eficaces y eficientes en su gestión.

Venezuela, hoy en día, atraviesa por una crisis económica y social de difícil superación desde hace varios años y la que pareciera agravarse. Una de las probables causas de la crisis económica y social venezolana es la caída de su aparato productivo por el acceso restringido a las divisas necesarias para adquirir o reponer insumos básicos o para la compra de mercancías en la continuidad de sus operaciones cotidianas; ello se ha agravado por la falta de apoyo de las instituciones estatales para suministrar las divisas, por lo que recurren a otros medios como el mercado paralelo (con precios exorbitantes del dólar) que estimulan a la vez la inflación y la escasez y desabastecimiento de bienes y servicios.

A pesar de las adversidades, la creciente demanda de productos y el emprendimiento del sector privado han propiciado el surgimiento y sostenimiento de empresas dedicadas al sector comercial (entre ellas las ferreterías); empresas que contribuyen y apoyan al sector de la construcción como reflejo del grado de desarrollo en la infraestructura de un país. En estas circunstancias las ferreterías proyectan un potencial significativo con el objetivo de impulsar el crecimiento económico de un país; adicionalmente, y medido en términos de bienestar colectivo, las ferreterías son fuentes generadoras de empleo. En los últimos años el sector ferretero también ha sido afectado por la situación económica del país dada la escasez de productos para reponer inventarios, la regulación en los precios y otra serie de factores que dificultan su producción y rentabilidad.

El gobierno venezolano ante esta situación reiteradamente señala su oposición a las políticas de mercado y a la liberalización comercial, razón por la cual su actuación se ha caracterizado por las regulaciones. Como parte de esas regulaciones, en Venezuela, desde hace varios años, se han instrumentado un conjunto de leyes para la fijación o determinación de precios de los bienes y servicios ofertados en el territorio nacional con el fin de garantizar el acceso de la población a los bienes y servicios. Específicamente con la entrada en vigencia de la Ley de Costos y Precios Justos de 2011, derogada posteriormente por la Ley Orgánica de Precios Justos de 2014 que se modificó más tarde por la Ley Orgánica de Precios Justos -LOPJ- de 2015; dichas leyes propuestas con la finalidad de regular todos los mecanismos para la determinación de precios en los productos o servicios provistos en todas las relaciones establecidas entre sujetos de derecho público o privado, natural o jurídico, nacional o extranjero (comerciales, productivas, de prestación de servicio e importación), en todo el territorio nacional.

\section{Antecedentes y revisión de la literatura}

De acuerdo a la teoría económica existen diferentes formas de fijar el precio a un bien o servicio, destacando entre ellas: las condiciones de mercado (que se fundamentan en la demanda y en la competencia) y los niveles o estructura de costos (métodos del costo total, del producto y/o variable), los cuales ocupan un lugar predominante en el proceso de fijación de precios (Warren, Reeve y Duchac, 2010); al igual que las variaciones en el entorno legal e incertidumbre económica son presiones que al momento de establecer precios convierten al trabajo en una actividad más complicada para la organización, por lo que conducen a los involucrados a desviarse de los métodos acostumbrados de fijación de precios (Márquez, 2005). 
Por otro lado en cuanto al margen de ganancias de las empresas, componente básico de la fijación de precios regulado por la LOPJ, Stefani (2013) indica que en

un mercado sano el margen de ganancia que se desea, siempre estará sujeto al precio que el comprador esté dispuesto a pagar por un bien o servicio, pero nunca se debería ver restringido por lo que el Estado considere unilateralmente como margen justo. (p. 21)

Respecto a la estructura de costos, en Venezuela, el 07 de febrero de 2014 es publicada en la Gaceta Oficial Número 40.351 la Providencia Administrativa № 003/2014 mediante la cual se fijan los "criterios contables generales para la determinación de precios justos"; de uso obligatorio para los sujetos de aplicación de la LOPJ en la adecuación de sus estructuras de costos y para determinar sus precios justos.

Enloconcernientealestablecimientodeprecios por parte de los sujetos de aplicación, el 27 de octubre de 2015, es promulgada en la Gaceta Oficial Número 40.775 la Providencia Administrativa № 070/2015 mediante la cual "se regulan las modalidades para la determinación, fijación y marcaje de precios en todo el territorio nacional". No obstante, ambas providencias (003 y 070) y la LOPJ han sido ejes de múltiples debates entre el sector público y privado (empresarial y contable) debido a discrepancias deducidas de la interpretación del contenido de este marco legal.

Para Martínez (2014) las anteriores discordancias son producto de la 003, pues "excluye algunos gastos y costos presentes en los registros contables de las empresas" (p. 3); al mismo tiempo que transforma las normas, procedimientos y criterios contables de la contabilidad de costos convencional y de las Normas de Información Financiera aceptadas en Venezuela -VEN-NIF- para costear o valorar inventarios y determinar el costo de ventas, así como los tratamientos contables de los gastos operacionales.

Por ello muchos empresarios y entes de la actividad productiva venezolana -incluidas las empresas ferreteras- temen la merma de su rentabilidad por la exclusión de algunos costos tales como gastos de distribución, gastos financieros y una parte importante de los gastos operativos (gastos ajenos a la producción) de su estructura de costos para la determinación de sus precios de acuerdo a las disposiciones de ambas providencias. Además temen por el desconocimiento o la incorrecta aplicación de las normativas administrativas, contables $y$ procedimientos por parte de los funcionarios encargados de fiscalizar el cumplimiento tanto de la LOPJ como de las providencias administrativas. Con base en ello afirma Semtei (2014) que la ley castiga la productividad, existiendo riesgos de que la misma sea aplicada a discrecionalidad del inspector o del funcionario de la Superintendencia Nacional para la Defensa de los Derechos Socioeconómicos -SUNDDE- encargado de hacer las fiscalizaciones.

Respecto a la función fiscalizadora de la LOPJ -y sus procesos de actualización de precios controlados- Álvarez (2014) indica que si los elementos del costo aumentan de modo permanente pero los precios permanecen congelados o sin actualización llegará un momento en que los costos superen el precio regulado, por lo que ni siquiera las empresas de la economía solidaria podrían producir. Ciertamente cuando la rigidez de los controles de precio produce pérdidas para las empresas se desestimula la producción y reaparecen los problemas de escasez, caldo de cultivo perfecto para que surjan las pérfidas prácticas del acaparamiento y la especulación. De ahí la importancia de examinar periódicamente la estructura de costos y mantener actualizados los precios, así como los criterios por los cuales se fijan. Si esto es considerado por los administradores de ley y se actúa de manera consciente, flexible y oportuna, no se castigará la producción y no se generará escasez ni acaparamiento o especulación.

Por lo anterior, según el Centro de Investigación y Consultoría Empresarial CAPEFI, la LOPJ puede ser vista como un desafío o una oportunidad para que las empresas formalicen sus procedimientos contables y a la vez puedan diagnosticar con mayor precisión las ganancias o pérdidas por las ventas de los productos y/o servicios ofrecidos. En este sentido Morillo (2004) expone que, aunque el nivel de precios no pueda ser fijado por la empresa debido a restricciones establecidas por diversos factores entre ellos el Estado, a las empresas les favorece conocer qué 
margen de ganancia perciben por cada uno de sus productos $-\mathrm{y}$ su estructura de costos- con la finalidad de seguir fabricándolos o dedicarse a la elaboración de otros para mantenerse en el ramo o cambiar de negocio.

\section{Objetivo de la investigación}

Ante el panorama descrito, y la revisión de literatura efectuada, la presente investigación está orientada a evaluar el efecto de la fijación de precios de las ferreterías ubicadas en el municipio Campo Elías del Estado Mérida (Venezuela) en el marco de la LOPJ de 2015. Específicamente se estudiaron los procedimientos de estructuración de costos para la determinación y fijación de precios en los productos comercializados, en virtud de las diferencias existentes entre los lineamientos para la estructuración de costos y la fijación de precios establecidos por la contabilidad de costos convencional y la enmarcada en la LOPJ y en sus providencias 003/2014 y 070/2015.

\section{Metodología}

Para alcanzar el objetivo de investigación trazado se diseñó una investigación no experimental bajo un enfoque cuantitativo de alcance analítico, previo a una fase exploratoria y descriptiva. La población estuvo conformada por 40 empresas dedicadas a las actividades comerciales del sector ferretero, ubicadas en el registro del Servicio Autónomo Municipal de Administración Tributaria del municipio Campo Elías del Estado Mérida. Debido a que el tamaño de la población es reducido se omitieron métodos de muestreo, lo cual equivalió a la práctica de un censo con una tasa de respuesta del $40 \%$; pues un grupo de estas empresas no pudieron ser ubicadas o contactadas por estar posiblemente inactivas (puertas cerradas) al momento de realizar la recolección de datos.

En aras de obtener el mayor grado de objetividad en la recolección de datos se utilizaron varias técnicas. La primera técnica de recolección de datos utilizada fue la revisión documental donde se recolectó información sobre los sujetos de estudio, contenida en documentos elaborados por personal de la empresa o terceras personas (estados financieros). Posteriormente, en la fase de investigación de campo, se realizó una encuesta estructurada o formalizada (apoyada en un cuestionario); este instrumento fue validado en su contenido mediante un juicio de expertos, lo que permitió calcular el coeficiente de proporción de rangos y encontrar una elevada concordancia de juicio en torno al ajuste del contenido del instrumento con las variables de estudio $(0,772)$.

\section{Resultados y discusión}

A partir del diseño metodológico planteado se halló que, pese a la gran diversidad de productos comercializados -concentrados en los equipos y materiales de electricidad-, las herramientas de construcción, las pinturas y accesorios, y los materiales de plomería (figura 1) se han reducido en los últimos años de acuerdo a lo que manifestó la mayoría de empresarios encuestados. Ello es producto de la baja capacidad de la reposición de inventario que a la vez es consecuencia, primero, del acceso limitado y casi inadmisible a las divisas para la adquisición de productos importados o fabricados por sus proveedores con componentes importados y segundo por los aumentos constantes de sueldos mínimos decretados por el ejecutivo nacional; esto no solo conduce a la reducción de la capacidad de compra de la empresa al restringir su capital de trabajo, sino también a la reducción y -en algunos casosa la suspensión de operaciones de las empresas proveedoras e importadoras de los productos mencionados por no soportar la carga laboral. Esto último es determinante ante la condición de microempresas de la mayoría de empresas estudiadas (figura 1). 


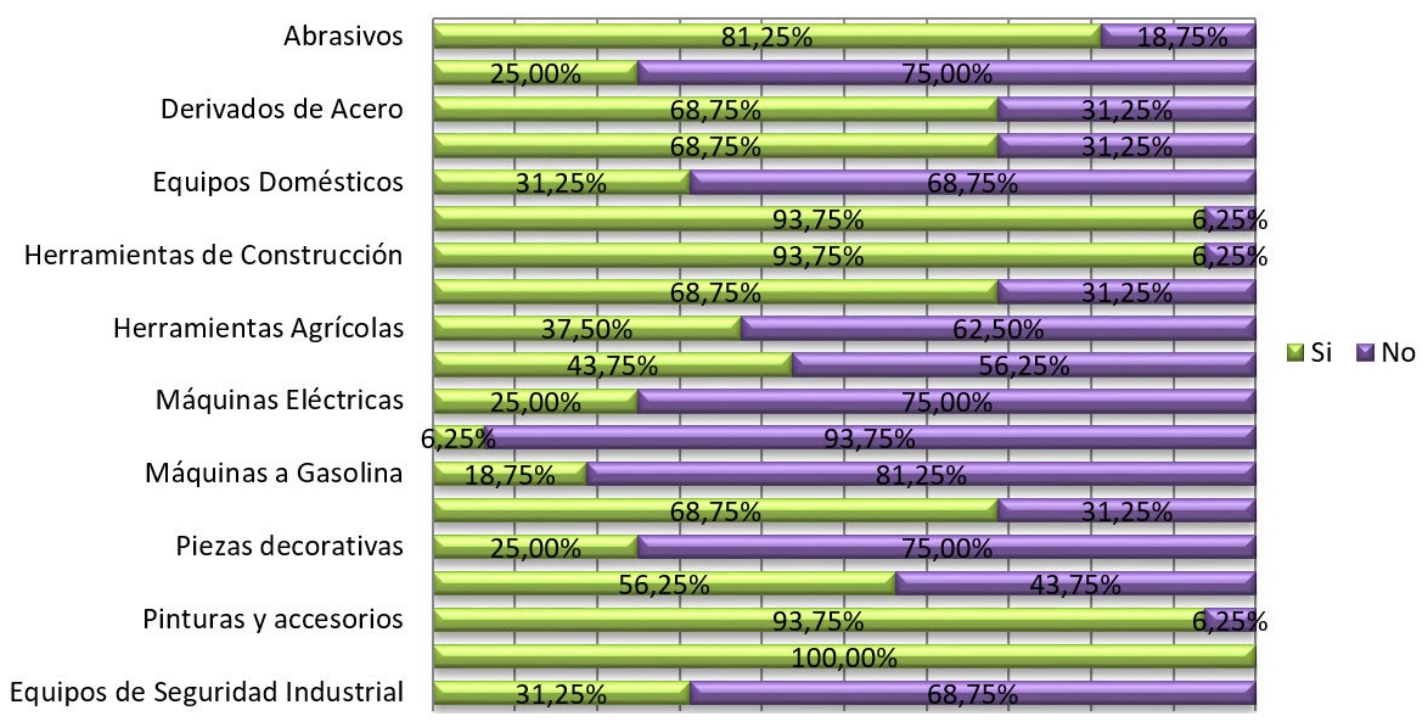

Figura 1. Productos comercializados por proporción de ferreterías. Fuente: elaboración propia por parte de los autores.

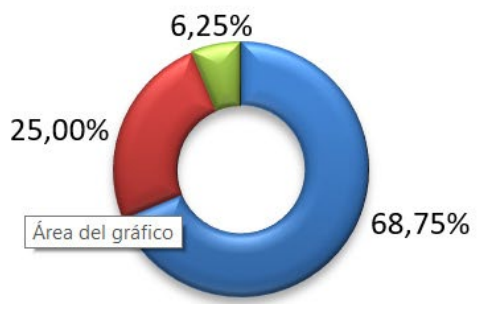

$\square$ Entre 1 y 4 trabajadores (Microempresa)
$\square$ Entre 5 y 20 trabajadores (Pequeña Empresa)
$\square$ Entre 21 y 100 trabajadores (Mediana Empresa)
$\square$ Mas de 100 (Empresa Grande)

Figura 2. Cantidad de empleados que labora en las empresas estudiadas. Fuente: elaboración propia por parte de los autores.

Sobre la estructura de costos, el $100 \%$ de las empresas estudiadas consideran como costo el valor de compra del producto que comercializan (costos según factura de la mercancía comprada) al momento de fijar los precios en caso de que el fabricante no fije el precio máximo de venta al público - PMVP- del producto tal como lo establece la Providencia Administrativa № $070 / 2015$. Los impuestos no recuperables o transferibles al consumidor son solo considerados por el $12,5 \%$ de las empresas, mientras que los costos de transporte, fletes o traslados en compras son considerados por el 81,25\% de empresas; el $12,75 \%$ de las empresas omiten este último costo por desconocimiento de la Providencia Administrativa № 003/2014 y de los métodos de prorrateo o asignación de costos por considerarlos como un costo mínimo o insignificante, pero en ningún caso se debe a inspecciones o sanciones por parte de la SUNDDE.

Ninguno de los ferreteros considera en el costo de sus productos vendidos aranceles y gastos de importación (aduanas) al no ser importadores de los productos que comercializan (tabla 1). Respecto a los costos de funcionamiento o gastos operativos se obtuvo que la mayor parte de ferreterías incurren en gastos de alquiler del local, gastos de publicidad, salarios de vendedores y almacenistas, y aportes parafiscales (figura 3); la mayor parte de empresarios no incorpora dichos gastos operativos en atención a la Providencia Administrativa № $003 / 2014$, la cual solo permite incorporar el 12,5\% de esos gastos en la estructura de costos a los fines de calcular precios. 
Tabla 1. Costos, gastos y factores considerados para fijar el PMVP cuando no son fijados por el proveedor

\begin{tabular}{|c|c|c|c|c|c|}
\hline $\begin{array}{c}\text { Costos } \\
\text { y gastos }\end{array}$ & Considerado & $\begin{array}{c}\text { No } \\
\text { Considerado }\end{array}$ & Factores & Considerado & $\begin{array}{c}\text { No } \\
\text { Considerado }\end{array}$ \\
\hline $\begin{array}{l}\text { Costos según factura de la } \\
\text { mercancía comprada }\end{array}$ & $100 \%$ & - & $\begin{array}{l}\text { Costo del producto }+ \\
\text { porcentaje de ganancia }\end{array}$ & $100 \%$ & - \\
\hline $\begin{array}{l}\text { Costos de transporte, fletes o } \\
\text { traslados }\end{array}$ & $87,50 \%$ & $12,50 \%$ & $\begin{array}{l}\text { Nivel de precios de la } \\
\text { competencia }\end{array}$ & $43,75 \%$ & $56,25 \%$ \\
\hline $\begin{array}{l}\text { Impuestos no recuperables o } \\
\text { transferibles }\end{array}$ & $18,75 \%$ & $81,25 \%$ & Nivel de demanda & $43,75 \%$ & $56,25 \%$ \\
\hline $\begin{array}{l}\text { Aranceles y gastos de } \\
\text { importación }\end{array}$ & - & $100 \%$ & $\begin{array}{l}\text { Poder adquisitivo } \\
\text { o disposición del } \\
\text { comprador }\end{array}$ & $18,75 \%$ & $81,25 \%$ \\
\hline Arrendamientos de locales & $37,50 \%$ & $62,50 \%$ & $\begin{array}{l}\text { Niveles de eficiencia } \\
\text { de la empresa }\end{array}$ & $25 \%$ & $75 \%$ \\
\hline Publicidad & $31,25 \%$ & $68,75 \%$ & Tipo de clientes & $43,75 \%$ & \\
\hline $\begin{array}{l}\text { Salarios de vendedores y } \\
\text { almacenistas }\end{array}$ & $31,25 \%$ & $68,75 \%$ & $\begin{array}{l}\text { (fidelidad y volumen de } \\
\text { compra) }\end{array}$ & & $56,25 \%$ \\
\hline $\begin{array}{l}\text { Salario de personal } \\
\text { administrativo }\end{array}$ & $31,25 \%$ & $68,75 \%$ & & & \\
\hline $\begin{array}{l}\text { Aportes parafiscales (SSO, } \\
\text { INCES entre otros) }\end{array}$ & $37,50 \%$ & $62,50 \%$ & & & \\
\hline
\end{tabular}

Fuente: elaboración propia por parte de los autores.

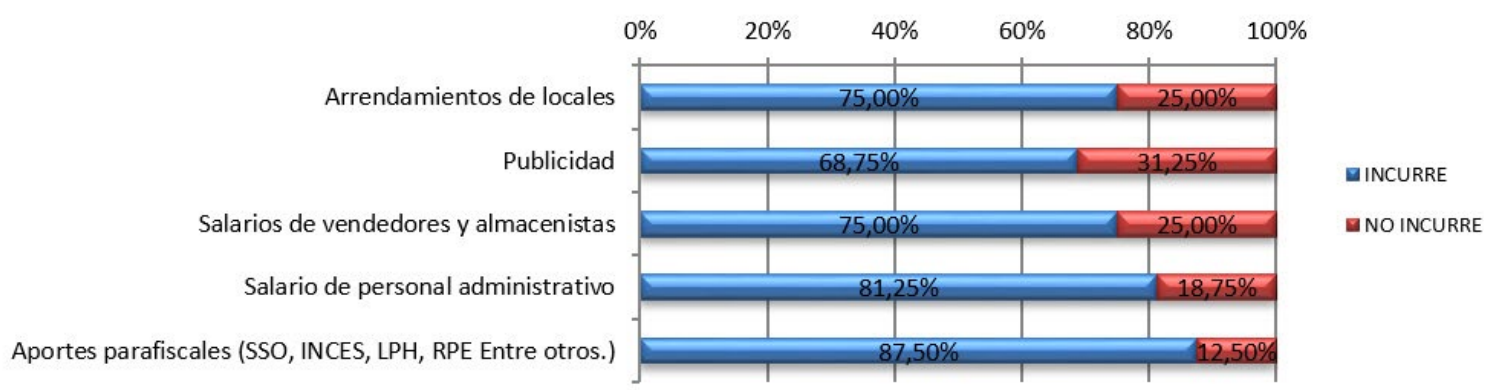

Figura 3. Gastos operativos incurridos por proporción de ferreterías. Fuente: elaboración propia por parte de los autores.

En cuanto al conocimiento o cálculo de las estructuras de costos por cada tipo de producto que comercializan, el $75 \%$ indicaron conocerlo; las restantes empresas evidencian un manejo de operaciones mercantiles y financieras insuficiente en lo relativo a los costos de las ventas como información básica para planificar y controlar la rentabilidad de cualquier organización con fines de lucro.

Se encontró que de las empresas que conocen la estructura de costos por tipo de producto, más del $83 \%$ la elaboran según la LOPJ (a través de las providencias administrativas 003/2014 y
070/2015); esto muestra preocupación por temas legislativos al ser sujetos de aplicación que desarrollan actividades económicas en el territorio nacional y preocupación por el manejo de las operaciones de sus negocios a fin de evitar multas y sanciones por parte del ente encargado y supervisor de la SUNDDE, pese a que solo el 25 $\%$ de las empresas estudiadas considera que la normativa legal ha realizado una contribución en la organización de su empresa y a la optimización contable y financiera de su negocio; lo anterior, frente a la gran mayoría que opina lo contrario $(56,25 \%)$. 
Respecto a los costos excluidos de la estructura por la Providencia Administrativa № 003/2014, que han sido parte integral y considerados por la contabilidad de costos convencional (contribuciones parafiscales, costos de fletes sobre ventas y compras, dealmacenamiento y la totalidad de gastos operativos), es importante destacar que solo el $69 \%$ de los ferreteros conoce esa exclusión. Esta misma proporción de empresarios manifestó como una de sus principales inconformidades la exclusión de la estructura de costos, los intereses y gastos por préstamos bancarios para la comercialización del producto, el costo de distribución, las contribuciones parafiscales y los efectos inflacionarios en el valor de los inventarios relacionados con los costos de reposición; ellos consideran que todas estas omisiones amenazan la continuidad de operaciones de la empresa, por lo que no pueden ser obviadas.

Sobre el marcaje y fijación de precios, más de la mitad de los precios (PMVP) de artículos ofertados por las ferreterías encuestadas no son fijados y marcados o etiquetados por su proveedor como, por ejemplo: las cerámicas y baldosas; los derivados del acero, del hierro; los equipos domésticos; las lámparas; las máquinas eléctricas, a gasoil y a gasolina; los materiales de construcción y las piezas decorativas. Lo anterior, a diferencia de otros grupos de productos que si presentan el PMVP al momento de ser comprados a los proveedores (por ejemplo, abrasivos, equipos y materiales de electricidad, de seguridad, herramientas tanto de construcción, eléctricas y agrícolas, piezas sanitarias y materiales de plomería).

Ahora bien, se evidencia cierto incumplimiento legal por parte de los sujetos de aplicación en lo que a la Providencia Administrativa № 070/2015 se refiere. Esto podría generar que a lo largo de la cadena de comercialización, desde la fábrica o importadora hasta las casas detallistas, los productos sean adulterados al ser removidas las etiquetas del PMVP por los miembros de la cadena de comercialización -especialmente aquellos productos cuyos precios no pueden ser rotulados sino etiquetados-; incluso algunas veces se modifica o se remueve el PMVP del cuerpo del bien, lo cual no permite al consumidor final observarlo al adquirirlo. Tales acciones conducen a razonar sobre la existencia de prácticas deshonestas por parte de los integrantes de la cadena de comercialización para aumentar el precio de venta de la mercancía y así presumiblemente obtener mayor margen de ganancia en sus operaciones, lo que amerita actividades de fiscalización por parte de los entes competentes.

De los factores considerados al momento de fijar el precio de los productos ofrecidos en el mercado ferretero, los datos recolectados y analizados reflejan una marcada tendencia hacia la fijación de precios en función de variables múltiples (como, por ejemplo, los niveles de la demanda, las acciones de la competencia y productos sustitutos, el poder adquisitivo del consumidor y los niveles de eficiencia de la misma empresa) en lugar de una sola como lo es el nivel de costo tal como lo establece la LOPJ y sus providencias administrativas (tabla 1). Específicamente sobre las metodologías, técnicas y procedimientos, y demás factores considerados para fijar el precio de los productos con mayor rotación o importancia para la empresa $-y$ cuyos PMVP no son fijados por su fabricante o importador- los empresarios consideran factores como la demanda, la eficiencia entre otros elementos y metodologías propias en la competencia de libre mercado, lo que los pone al margen de la LOPJ y en peligro de las sanciones por infracciones y delitos.

En relación con la consideración o cuantificación del costo del flete sobre compras al costo unitario de la mercancía y de otros conceptos (gastos de distribución, ventas y mercadeo, publicidad, salarios de vendedores y almacenistas, gastos de administración, impuestos no recuperables o transferibles, arrendamientos de locales, salario de personal administrativo y aportes parafiscales) no permitidos por la Providencia Administrativa № 070/2015 implícitamente, ya que solo considera el costo unitario según factura de compra más un $30 \%$ de margen de ganancia máximo para fijar el PMVP (artículo 31 de la LOPJ), se interpreta que la Providencia Administrativa № 003/2014 no es aplicable a las empresas comercializadoras (mayoristas o detallistas); de ahí que se excluye dentro de su estructura de costos los gastos ajenos a la producción, pues solo son considerados para fabricantes o prestadores de servicios hasta un máximo de $12,5 \%$ respecto al costo de producción (según el artículo 2, numeral 12, de la Providencia Administrativa № 003/2014). 
Respecto al margen de comercialización utilizado al momento de establecer el precio de los productos comercializados y cuyo PMVP no es fijado por el fabricante, la mayoría de empresas utiliza márgenes inferiores al $30 \%$ como margen bruto de utilidad en ventas tal como lo establece la LOPJ; ninguno de los ferreteros utiliza márgenes inferiores de comercialización del $10 \%$ ni superiores al $45 \%$, pero si una considerable porción $(12,5 \%)$ utilizan un margen de ganancia por unidad vendida entre el $31 \mathrm{y}$ el $45 \%$ al momento de fijar sus precios. Lo que evidencia, nuevamente, riesgo de ser sancionados por desacato a la normativa al margen máximo de ganancia debido a la apremiante necesidad de recuperar sus inversiones (expresadas en el costo de los productos para ser comercializados); además de tener que soportar, en su utilidad bruta en ventas, un porcentaje representativo de gastos operativos o de funcionamiento.

Frente a la rentabilidad de las ferreterías, en el marco de la LOPJ, la misma se ha reducido en la totalidad de empresas estudiadas. Esto es producto de las mismas restricciones impuestas para el margen máximo de ganancia (30\%), el cual no permite cubrir los grandes y sucesivos incrementos del costo de la mercancía y los elevados gastos operativos del negocio (nómina, alquileres, impuestos, servicios de asesoría entre otros), lo que ocasiona que tanto los márgenes de utilidad bruta sobre venta como la utilidad operativa hayan resultado fuertemente oprimidos. Igualmente la rentabilidad se mostró deprimida por la reducción en el nivel de ventas en las ferreterías estudiadas, producto a la vez de los altos niveles de inflación que devora no solo el poder adquisitivo de los consumidores de productos ferreteros sino el margen de utilidad por la venta de productos y la posibilidad de reposición del inventario, desestimulando así la actividad comercial; asimismo, la escasez en el mercado mayorista contribuyó con la reducción del nivel de ventas.

Los menores márgenes de utilidad operativa percibidos por los ferreteros $(23,86 \%)$ se concentran en el grupo de productos derivados del hierro (figura 4). Los productos por los que obtienen un margen de ganancia más alto (30 \%) son los equipos domésticos, las máquinas eléctricas y las máquinas a gasoil, pues son los que precisamente presentan un menor grado de comercialización (figura 1). Este menor grado de comercialización es atenuado en cierta parte por el margen de ganancia obtenido, por lo que se deduce que la rentabilidad de las empresas se relaciona directamente con los niveles de ventas de los productos comercializados. De igual manera se deduce que los constantes controles de precios y de márgenes de ganancia que podrían contribuir con la contracción o merma de utilidades no han podido eliminar el atractivo o rentabilidad para la actividad económica desarrollada por las ferreterías estudiadas o a la vez causar un estrangulamiento o cese de actividades en el sector luego de la rigurosidad en la aplicación de la LOPJ por parte de los sujetos fiscalizadores, siempre y cuando que los niveles de ventas de algunos rubros se mantengan elevados.

Finalmente los factores que inciden en la rentabilidad de sus empresas tanto financiera como operativamente a juicio de los empresarios se encontraron en una mayor proporción en las condiciones del sector donde se desenvuelven, en la eficiencia empresarial y en las fuentes de financiamiento (figura 5). Otros factores menos considerados por los empresarios es la motivación al personal y la atención al cliente, factores clave en las operaciones medulares a partir de su impacto en la productividad de los empleados, reducción de costos y nivel de ventas.

De igual manera la metodología utilizada por los ferreteros para incrementar la rentabilidad operativa a partir del uso de los costos fijos (apalancamiento operativo), no es practicada por una considerable porción de empresas (60\%) debido al desconocimiento de su estructura de costos y al no estar perfectamente determinada. Lo anterior, apunta a señalar que dependiendo del conocimiento que los empresarios tengan de la estructura de costos adecuada y del apalancamiento operativo es posible utilizar esta estrategia en beneficio de la empresa. De hecho, solo una pequeña porción de los ferreteros practican un análisis de sensibilidad de sus inversiones y capital de trabajo en sus estructuras de costos, utilidades y rentabilidad. 


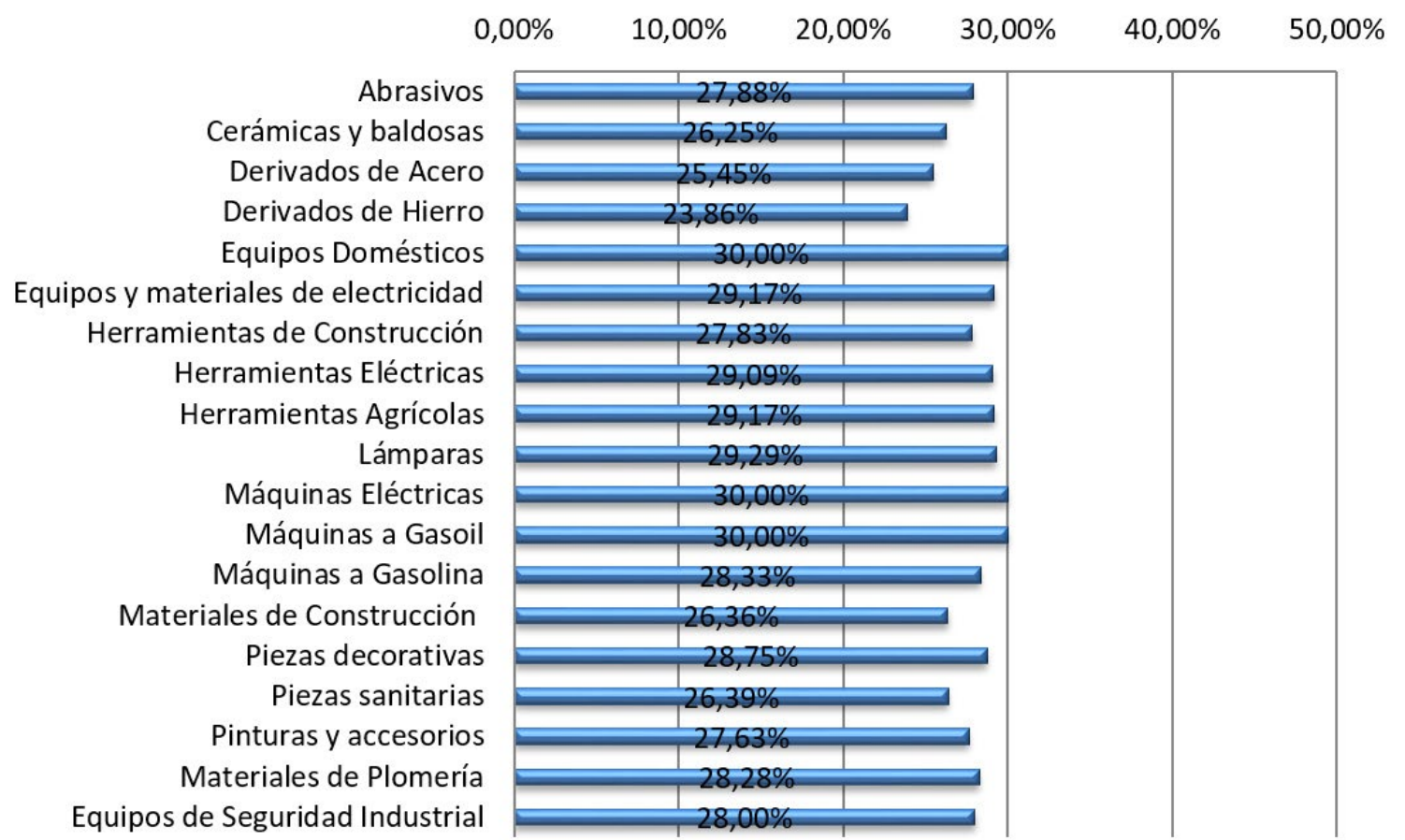

Figura 4. Margen de ganancia operativa promedio por grupo de productos comercializados. Fuente: elaboración propia por parte de los autores.

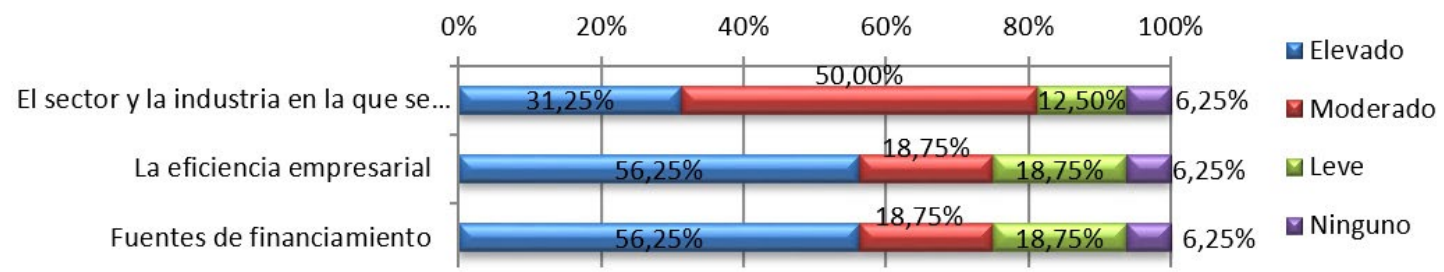

Figura 5. Factores que inciden en la rentabilidad por proporción de ferreterías. Fuente: elaboración propia por parte de los autores.

\section{Conclusiones}

A partir de las observaciones realizadas y de la encuesta practicada se evidenciaron algunas equivocaciones o inexactitudes en los procedimientos de aplicación de la LOPJ y sus providencias administrativas por los sujetos de estudio en cuanto a sus modelos de estructuras de costos y métodos de asignación de precios, en sus operaciones medulares (compra y venta de mercancía). Estas debilidades llevan a prácticas que afectan la rentabilidad de sus negocios y al mismo tiempo aceleran la vulnerabilidad de esas empresas ante sanciones por el desconocimiento o desapego a la ley. Algunos de los incumplimientos legales por parte de los sujetos de aplicación en lo que a la Providencia Administrativa № 070/2015 se refiere tienen que ver con que más de la mitad de los precios (PMVP) de artículos ofertados por las ferreterías encuestadas no son fijados y marcados o etiquetados por su proveedor sino por la misma ferretería. Otro incumplimiento importante se halló en el margen de comercialización utilizado (superior al $30 \%$ ) por el $12,5 \%$ de los empresarios al momento de establecer el PMVP y que no es fijado por el fabricante, lo que involucra para el ferretero un alto riesgo de ser sancionado frente a la necesidad de recuperar sus inversiones en el costo de los productos para ser comercializados y de no generar pérdidas en el negocio. 
A través del análisis efectuado se formulan una serie de recomendaciones dirigidas tanto a la gerencia como al gobierno en el marco de la LOPJ para el desarrollo y mantenimiento de ventajas competitivas basadas principalmente en la reducción de costos y en la diferenciación del servicio, las cuales podrían coadyuvar a mejorar las operaciones desarrolladas por las ferreterías estudiadas. En primer lugar se recomienda la reducción de los costos, especialmente de los gastos operativos, calificados como gastos ajenos a la producción a partir del incremento de la eficiencia en las operaciones de la empresa, de la identificación de mejores formas de hacer las cosas que pudieran reducir costos y agregar mayor valor a los clientes y a la empresa.

Por tal motivo, para la gerencia de las empresas estudiadas la información de costos debe ser considerada relevante; para ello deben conocer su estructura de costos por producto comercializado no solo para la toma de decisiones sino para el control eficiente de los costos, orientado a la reducción de los mismos y al diseño y mantenimiento de ventajas competitivas; esto es urgente para el $25 \%$ de los empresarios que manifestaron desconocer su estructura de costos. En este caso se sugiere la capacitación y actualización de los profesionales que ejercen cargos administrativos en las ferreterías sobre procedimientos expeditos de asignación de costos (tal es el caso de la asignación de costos por fletes sobre compras entre otros), los cuales son omitidos por parte de una considerable porción de empresarios. Se sugiere la realización de cursos y talleres sobre procedimientos de acumulación y asignación de costos para lograr una adecuada preparación y actualización en relación con estas materias tan cambiantes como las jurídicas.

Ya que los niveles de rentabilidad y de supervivencia de la empresa dependen de los niveles de venta o volúmenes de producción ante las regulaciones del margen máximo de ganancia, y a que la mayoría de empresarios están de acuerdo con las regulaciones y están dispuestos a continuar sus operaciones con un margen máximo del $30 \%$ siempre que los volúmenes de ventas sean altos, se sugiere a las ferreterías del municipio Campo Elías continuar haciendo esfuerzos por mantener los niveles de venta. En este caso las actividades de diferenciación y de posicionamiento en el mercado podrían contribuir a mantener e incrementar los niveles de venta. Estas actividades de diferenciación podrían consistir en esfuerzos de calidad en el servicio que se refleja en una excelente atención al cliente, asesoría, actitudes de empatía, confiabilidad y responsabilidad.

Aunque estos esfuerzos de incrementar el nivel de ventas pueden ser infructuosos o muy difíciles dado los niveles de recesión económica, de escaso poder adquisitivo de los consumidores y de desempleo que retraen los niveles de demanda y por lo tanto los niveles de actividad de las empresas. Por ello se sugiere al gobierno el control de la inflación y la reactivación del aparato productivo, los cuales coadyuven efectivamente ante la recesión económica. Estas serían las condiciones ideales de operación con un marco regulatorio que incluya un margen máximo de intermediación del $60 \%$ para todos los intermediarios o integrantes de la cadena de comercialización y una regulación individual de ganancia máxima de cada uno de ellos del $30 \%$.

Dada la reducción de variedad de productos ofertados por las empresas ferreteras se sugiere al gobierno el desarrollo de procedimientos expeditos para la administración de divisas y la estimulación de las actividades de manufactura de materiales y bienes para la construcción, pues son estas las principales causas que han disminuido la oferta de bienes de la construcción en toda la cadena de valor industrial (es decir, desde el productor e importador hasta su venta al detal).

De igual manera en el ambiente inflacionario cuyo porcentaje (30\% de utilidad) no permite reponer inventario, ya que los costos de reposición son superiores al PMVP de los productos vendidos, se sugiere al poder legislativo nacional establecer el marco legal en los procesos de fijación de precios y ajustar lo contenido en la Providencia Administrativa № 003/2014; al contemplar dentro de los procesos de fijación de precio la variable inflacionaria en el comportamiento de los precios de los bienes a nivel de productor e importador. Además se recomienda orientar el contenido en la misma providencia no solo al sector manufacturero y de servicio, ya que la misma no es aplicable al sector comercial al solo considerar los costos de adquisición de los productos como costos de producción y los demás costos como gastos ajenos 
(supeditados en algunas oportunidades al 12,5\% del costo de producción y en otras simplemente son omitidos) puesto que precisamente los servicios adicionales que prestan las ferreterías incluyen una gran cantidad de costos asociados a la gestión empresarial, al servicio al cliente y a las entregas (gastos administrativos y de ventas). En virtud de ello se considera pertinente que a las empresas comercializadoras se les deba elevar el margen porcentual de los gastos ajenos a producción dentro de su estructura de costos de sus productos para cubrir sus gastos operativos o de funcionamiento, respaldando este porcentaje con un análisis vertical del estado de ganancias y pérdidas del mes inmediatamente anterior tal como es considerado el $12,5 \%$ de gastos ajenos para las manufactureras o prestadoras de servicio; y de esta manera al momento de establecer un PMVP se pueda recuperar parte de los gastos del ejercicio o del período, así como permitir una rentabilidad justa.

Finalmente producto del constante incumplimiento legal por parte de los sujetos de aplicación en lo que a la Providencia Administrativa № 070/2015 se refiere, en especial en el caso del marcaje del PMVP por parte del proveedor que conduce a constantes riesgos de remoción de precios y demás prácticas deshonestas por parte de los integrantes de la cadena de comercialización, se requieren mayores esfuerzos en las actividades de fiscalización por parte de la SUNDDE.

Debido al elevado grado de confidencialidad y hermetismo que impera hoy en el sector es importante desarrollar una investigación con un nutrido enfoque cuantitativo donde se pueda medir porcentualmente el margen de ganancia, el nivel de costos y el comportamiento del nivel de ventas de las empresas de acuerdo a los parámetros establecidos por la LOPJ vigente. Igualmente se pueden desarrollar estudios sobre las percepciones empresariales en ambientes de precios y ganancias controladas (es decir, sobre el grado de aceptación, incentivos y riesgos percibidos por el empresariado) de las cuales dependen los planes de permanencia y expansión de tan importante actividad económica.

\section{Referencias}

Álvarez, V. (2014). El impacto económico de la Ley de Precios Justos. Recuperado de https://www. aporrea.org/endogeno/a183234.html.

Márquez, A. (2005). Una mirada integral a la decisión de precios de la organización. Visión Gerencial, 4(1), 42-52.

Martínez, J. (2014). La Ley de Precios y las NIIF en Venezuela (VEN-NIF). Recuperado de https:// niifpymes-venezuela.blogspot.com/2014/02/laley-de-precios-y-las-niif-en.html.

Morillo, M. (2014). Estructura de costos y presentación del estado de costos de producción y ventas. Mérida, Venezuela: Universidad de Los Andes.

Semtei, E. (2014). Más sobre la Ley de Precios Justos. Recuperado de https://www.reportero24. com/2014/03/03/eduardo-semtei-mas-sobre-laley-de-precios-justos/.

Stefani, R. (2013). Comentarios sobre la inconstitucionalidad de la Ley de Costos y Precios Justos. Ulpiano, 5, 219-242.

Warren, C., Reeve, J. y Duchac, J. (2010). Contabilidad administrativa. Ciudad de México, México: Cengage Learning Editores S.A. de C.V. 